\title{
A Novel Context-Sensitive Semisupervised SVM Classifier Robust to Mislabeled Training Samples
}

\author{
Lorenzo Bruzzone, Senior Member, IEEE, and Claudio Persello, Student Member, IEEE
}

\begin{abstract}
This paper presents a novel context-sensitive semisupervised support vector machine $\left(\mathrm{CS}^{4} \mathrm{VM}\right)$ classifier, which is aimed at addressing classification problems where the available training set is not fully reliable, i.e., some labeled samples may be associated to the wrong information class (mislabeled patterns). Unlike standard context-sensitive methods, the proposed $\mathrm{CS}^{4} \mathrm{VM}$ classifier exploits the contextual information of the pixels belonging to the neighborhood system of each training sample in the learning phase to improve the robustness to possible mislabeled training patterns. This is achieved according to both the design of a semisupervised procedure and the definition of a novel contextual term in the cost function associated with the learning of the classifier. In order to assess the effectiveness of the proposed $\mathrm{CS}^{4} \mathrm{VM}$ and to understand the impact of the addressed problem in real applications, we also present an extensive experimental analysis carried out on training sets that include different percentages of mislabeled patterns having different distributions on the classes. In the analysis, we also study the robustness to mislabeled training patterns of some widely used supervised and semisupervised classification algorithms (i.e., conventional support vector machine (SVM), progressive semisupervised SVM, maximum likelihood, and $k$-nearest neighbor). Results obtained on a very high resolution image and on a medium resolution image confirm both the robustness and the effectiveness of the proposed $\mathrm{CS}^{4} \mathrm{VM}$ with respect to standard classification algorithms and allow us to derive interesting conclusions on the effects of mislabeled patterns on different classifiers.
\end{abstract}

Index Terms-Context-sensitive classification, image classification, mislabeled training patterns, noisy training set, remote sensing, semisupervised classification, support vector machines (SVMs).

\section{INTRODUCTION}

$\mathbf{T}$ HE CLASSIFICATION of remote sensing images is often performed by using supervised classification algorithms, which require the availability of labeled samples for the training of the classification model. All these algorithms are sharply affected from the quality of the labeled samples used for training the classifier, whose reliability is of fundamental importance for an adequate learning of the properties of the investigated scene (and, thus, for obtaining accurate classification maps). In supervised classification, the implicit assumption is that all labels associated with training patterns are correct. Unfortunately, in many real cases, this assumption does not hold,

Manuscript received February 15, 2008; revised July 25, 2008 and October 2, 2008. First published May 12, 2009; current version published June 19, 2009.

The authors are with the Department of Information Engineering and Computer Science, University of Trento, 38050 Trento, Italy (e-mail: lorenzo. bruzzone@ing.unitn.it; claudio.persello@disi.unitn.it).

Color versions of one or more of the figures in this paper are available online at http://ieeexplore.ieee.org.

Digital Object Identifier 10.1109/TGRS.2008.2011983 and small amounts of training samples are associated with a wrong information class due to errors occurred in the phase of collection of labeled samples. Labeled samples can be derived by the following: 1) in situ ground truth surveys; 2) analysis of reliable reference maps; or 3) image photointerpretation. In all these cases, mislabeling errors are possible. During the ground truth surveys, mislabeling errors may occur due to imprecise geolocalization of the positioning system; this leads to the association of the identified land-cover label with a wrong geographic coordinate and, thus, with the wrong pixel (or region of interest) in the remotely sensed image. Similar errors may occur if the image to be classified is not precisely georeferenced. When reference maps are used for extracting label information, possible errors present in the maps propagate to the training set. The case of image photointerpretation is also critical, as errors of the human operator may occur, leading to a mislabeling of the corresponding pixels or regions.

Mislabeled patterns bring distort (wrong) information to the classifier (in this paper, we call them noisy patterns). The effect of noisy patterns in the learning phase of a supervised classifier is to introduce a bias in the definition of the decision regions, thus decreasing the accuracy of the final classification map. We can expect two different situations with respect to the distribution of noisy samples in the training set: 1) mislabeled samples may be uniformly distributed over all considered classes, or 2) mislabeled patterns can specifically affect one or a subset of the classes of the considered classification problem. The two different situations result in a different impact on the learning phase of the classification algorithms. Let us analyze the problem according to the Bayes decision theory and to the related estimates of class conditional densities (likelihood) and class prior probabilities (priors) [1]. If noisy samples are uniformly distributed over classes, the estimations of class conditional densities result corrupted, while the estimations of prior probabilities are not affected from the presence of mislabeled patterns. On the contrary, if noisy samples are not uniformly distributed over classes, both the estimations of prior probabilities and of class conditional densities are biased from mislabeled patterns. Therefore, we expect that supervised algorithms, which (explicitly or implicitly) consider the prior probabilities for the classification of a generic input pattern (e.g., Bayesian classifier and $k$-nearest neighbor $(k-\mathrm{NN})$ [1]-[3]), are more sensitive to unbalanced noisy sample distributions over classes than other algorithms that take into account only the class conditional densities (e.g., maximum likelihood (ML) [1], [2]).

In this paper, we address the aforementioned problems by the following: 1) presenting a novel context-sensitive 
semisupervised support vector machine $\left(\mathrm{CS}^{4} \mathrm{VM}\right)$ classification algorithm, which is robust to noisy training sets, and 2) analyzing the effect of noisy training patterns and of their distribution on the classification accuracy of widely used supervised and semisupervised classifiers.

The choice of developing a support vector machine (SVM)based classifier is related to the important advantages that SVMs exhibit over other standard supervised algorithms [4]-[8]: 1) relatively high empirical accuracy and excellent generalization capabilities; 2) robustness to the Hughes phenomenon [9]; 3) convexity of the cost function used in the learning of the classifier; 4) sparsity of the solution; and 5) possibility to use the kernel trick for addressing nonlinear problems. In particular, the generalization capability of SVM (induced by the minimization of the structural risk) gives to SVM-based classifiers an intrinsic higher robustness to noisy training patterns than other standard algorithms that are based on the empirical risk minimization principle. In this framework, we propose an SVM-based technique for image classification particularly developed to improve the robustness of standard SVM to the presence of noisy samples in the training set. The main idea behind the proposed $\mathrm{CS}^{4} \mathrm{VM}$ is to exploit the spatial context information provided by the pixel belonging to the neighborhood system of each training sample (which are called context patterns) in order to contrast the bias effect due to the possible presence of mislabeled training patterns. This is achieved by both a semisupervised procedure (aiming to obtain the semilabels for context patterns) and the definition of a novel contextual term in the cost function associated with the learning of the $\mathrm{CS}^{4} \mathrm{VM}$. It is worth noting that this use of the contextual information is completely different from that of traditional context-sensitive classifiers (see, e.g., [10]-[16]), where contextual information is exploited for regularizing classification maps in the decision phase.

Another important contribution of this paper is to present an extensive experimental analysis to investigate and compare the robustness to noisy training sets of the proposed $\mathrm{CS}^{4} \mathrm{VM}$ and of other conventional classifiers. In greater detail, we considered the (Gaussian) ML classifier (which is based on a parametric estimation of the class conditional densities and does not consider the prior probabilities of the classes), the $k$-NN classifier (which is based on a distribution-free local estimation of posterior probabilities that implicitly considers the class prior probabilities), the standard SVM classifier, and the progressive semisupervised SVM $\left(\mathrm{PS}^{3} \mathrm{VM}\right)$ [17]. The five considered classification algorithms were tested on two different data sets: 1) a very high resolution (VHR) multispectral image acquired by the Ikonos satellite and 2) a medium-resolution multispectral image acquired by the Landsat- 5 Thematic Mapper (TM). The experimental analysis was carried out, considering training sets that include different amounts of noisy samples having different distributions over the considered classes.

This paper is organized into six sections. Section II presents the proposed $\mathrm{CS}^{4} \mathrm{VM}$ technique. Section III describes the design of the experiments carried out with different classifiers. Sections IV and V illustrate the experimental results obtained on the Ikonos and Landsat data sets, respectively. Finally, Section VI, after discussion, draws the conclusion of this paper.

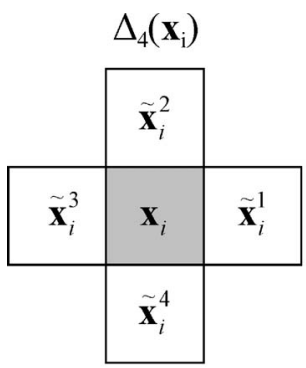

(a)

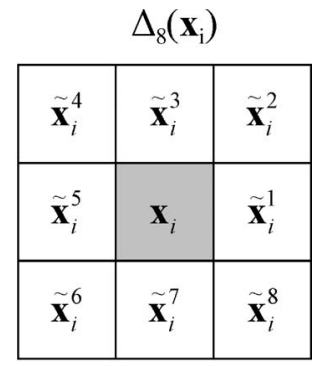

(b)
Fig. 1. Examples of neighborhood systems for the generic training pixel $\mathbf{x}_{i}$. (a) First-order system $\Delta_{4}\left(\mathbf{x}_{i}\right)$. (b) Second-order system $\Delta_{8}\left(\mathbf{x}_{i}\right)$.

\section{Proposed $\mathrm{CS}^{4} \mathrm{VM}$}

Let $\mathcal{I}$ denote a multispectral $d$-dimensional image of size $I \times J$ pixels. Let us assume that a training set $\mathcal{D}=\{\mathcal{X}, \mathcal{Y}\}$ made up of $N$ pairs $\left(\mathbf{x}_{i}, y_{i}\right)_{i=1}^{N}$ is available, where $\mathcal{X}=\left\{\mathbf{x}_{i} \mid \mathbf{x}_{i} \in \mathbb{R}^{d}\right\}_{i=1}^{N} \subset \mathcal{I}$ is a subset of $\mathcal{I}$ and $\mathcal{Y}=\left\{y_{i}\right\}_{i=1}^{N}$ is the corresponding set of labels. For the sake of simplicity, since SVMs are binary classifiers, we first focus the attention on the two-class case (the general multiclass case will be addressed later). Accordingly, let us assume that $y_{i} \in\{+1 ;-1\}$ is the binary label of the pattern $\mathbf{x}_{i}$. We also assume that a restricted amount $\delta$ of training samples $\mathbf{x}_{i}$ may be associated with wrong labels $y_{i}$, i.e., labels that do not correspond to the actual class of the considered pixel. Let $\Delta_{M}(\mathbf{x})$ represent a local neighborhood system (whose shape and size depend on the specific investigated image and application) of the generic pixel $\mathbf{x}$, where $M$ indicates the number of pixels considered in the neighborhood. Generally, $\Delta_{M}(\mathbf{x})$ is a first- or second-order neighborhood system (see Fig. 1). Let $\tilde{\mathcal{X}}=\left\{\tilde{\mathbf{x}}_{i}^{j} \mid \tilde{\mathbf{x}}_{i}^{j} \in \Delta_{M}\left(\mathbf{x}_{i}\right), \forall \mathbf{x}_{i} \in \mathcal{X}\right.$, $j=1, \ldots, M\}$ be the set of (unlabeled) context patterns $\tilde{\mathbf{x}}_{i}^{j}$ made up of the pixels belonging to the neighborhood $\Delta_{M}\left(\mathbf{x}_{i}\right)$ of the generic training sample $\mathbf{x}_{i}$. It is worth noting that adjacent training pixels belong to both $\mathcal{X}$ and $\tilde{\mathcal{X}}$.

The idea behind the proposed methodology is to exploit the information of the context patterns $\tilde{\mathcal{X}}$ to reduce the bias effect of the $\delta$ mislabeled training patterns on the definition of the discriminating hyperplane of the SVM classifier, thus decreasing the sensitivity of the learning algorithm to unreliable training samples. This is accomplished by explicitly including the samples belonging to the neighborhood system of each training pattern in the definition of the cost function used for the learning of the classifier. These samples are considered by exploiting the labels derived through a semisupervised classification process (for this reason, they are called semilabeled samples) [18]-[20]. The semilabeled context patterns have the effect to mitigate the bias introduced by noisy patterns by adjusting the position of the hyperplane. This strategy is defined according to a learning procedure for the proposed $\mathrm{CS}^{4} \mathrm{VM}$ that is based on two main steps: 1) supervised learning with original training samples and classification of the (unlabeled) context patterns and 2) contextual semisupervised learning based on both original labeled patterns and semilabeled context patterns according to a novel cost function. These two steps are described in detail in the following sections. 


\section{A. Step 1-Supervised Learning and Classification of Context Patterns}

In the first step, a standard supervised SVM is trained by using the original training set $\mathcal{D}$ in order to classify the patterns belonging to the neighborhood system of each training pixels. The learning is performed according to the soft-margin SVM algorithm, which results in the following constrained minimization problem:

$$
\left\{\begin{array}{l}
\min _{\mathbf{w}, b, \xi}\left\{\frac{1}{2}\|\mathbf{w}\|^{2}+C \sum_{i=1}^{N} \xi_{i}\right\} \\
y_{i} \cdot\left[\mathbf{w} \cdot \Phi\left(\mathbf{x}_{i}\right)+b\right] \geq 1-\xi_{i} \quad \forall i=1, \ldots, N \\
\xi_{i} \geq 0
\end{array}\right.
$$

where $\mathbf{w}$ is a vector normal to the separation hyperplane, $b$ is a constant such that $b /\|\mathbf{w}\|$ represents the distance of the hyperplane from the origin, $\Phi(\cdot)$ is a nonlinear mapping function, $\xi_{i}$ 's are slack variables that control the empirical risk (i.e., the number of training errors), and $C \in \mathbb{R}_{0}^{+}$is a regularization parameter that tunes the tradeoff between the empirical error and the complexity term (i.e., the generalization capability). The aforementioned minimization problem can be rewritten in the dual formulation by using the Lagrange optimization theory, which leads to the following dual representation:

$$
\begin{cases}\max _{\alpha}\left\{\sum_{i=1}^{N} \alpha_{i}-\frac{1}{2} \sum_{i=1}^{N} \sum_{j=1}^{N} y_{i} y_{j} \alpha_{i} \alpha_{j} k\left(\mathbf{x}_{i}, \mathbf{x}_{j}\right)\right\} & \\ \sum_{i=1}^{N} y_{i} \alpha_{i}=0 & \\ 0 \leq \alpha_{i} \leq C & \end{cases}
$$

where $\alpha_{i}$ 's are the Lagrange multipliers associated with the original training patterns $\mathbf{x}_{i} \in \mathcal{X}$, and $k(\cdot, \cdot)$ is a kernel function such that $k(\cdot, \cdot)=\Phi(\cdot) \Phi(\cdot)$. The kernel function is used for implicitly mapping the input data into a high-dimensional feature space without knowing the function $\Phi(\cdot)$ and still maintaining the convexity of the objective function [6]. Once $\alpha_{i}(i=1, \ldots, N)$ are determined, each context pattern $\tilde{\mathbf{x}}_{i}^{j}$ in the neighborhood system $\Delta_{M}\left(\mathbf{x}_{i}\right)$ of the training pattern $\mathbf{x}_{i}$ is associated with a semilabel $\tilde{y}_{i}^{j}$ according to

$$
\hat{\tilde{y}}_{i}=\operatorname{sgn}\left[\sum_{n=1}^{N} y_{n} \alpha_{n} k\left(\mathbf{x}_{n}, \tilde{\mathbf{x}}_{i}^{j}\right)+b\right] \forall \mathbf{x}_{n} \in \mathcal{X}, \forall \tilde{\mathbf{x}}_{i}^{j} \in \tilde{\mathcal{X}}
$$

where, given $f(\mathbf{x})=\sum_{i=1}^{N} y_{i} \alpha_{i} k\left(\mathbf{x}_{i}, \mathbf{x}\right)+b, b$ is chosen so that $y_{i} f\left(\mathbf{x}_{i}\right)=1$ for any $i$ with $0<\alpha_{i}<C$.

\section{B. Step 2-Context-Sensitive Semisupervised Learning}

Taking into account the semilabels (i.e., the labels obtained in the previous step) of the context patterns belonging to $\tilde{\mathcal{X}}$, we define the following novel context-sensitive cost function for the learning of the classifier:

$$
\Psi(\mathbf{w}, \xi, \psi)=\frac{1}{2}\|\mathbf{w}\|^{2}+C \sum_{i=1}^{N} \xi_{i}+\sum_{i=1}^{N} \sum_{j=1}^{M} \kappa_{i}^{j} \psi_{i}^{j}
$$

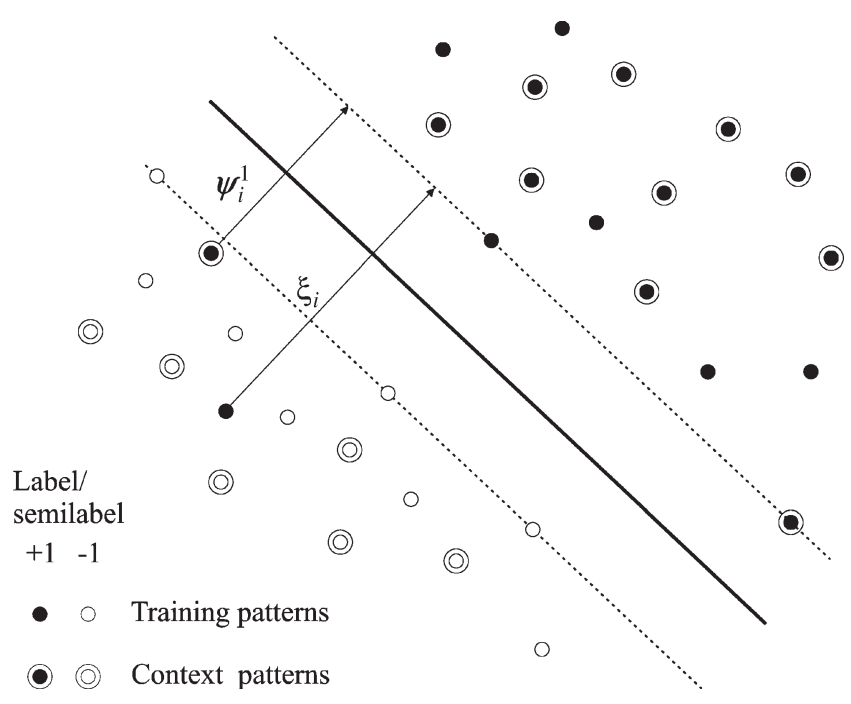

Fig. 2. Example of training and related context patterns in the kernel-induced feature space.

where $\psi_{i}^{j}$,s are context slack variables and $\kappa_{i}^{j} \in \mathbb{R}_{0}^{+}$are parameters that permit one to weight the importance of context patterns (see Fig. 2). The resulting constrained minimization problem associated with the learning of the $\mathrm{CS}^{4} \mathrm{VM}$ is the following:

$$
\left\{\begin{array}{cc}
\min _{\mathbf{w}, b, \xi, \psi} \Psi(\mathbf{w}, \xi, \psi) & \\
y_{i} \cdot\left[\mathbf{w} \cdot \Phi\left(\mathbf{x}_{i}\right)+b\right] \geq 1-\xi_{i} & \forall i=1, \ldots, N \\
\tilde{y}_{i}^{j} \cdot\left[\mathbf{w} \cdot \Phi\left(\tilde{\mathbf{x}}_{i}^{j}\right)+b\right] \geq 1-\psi_{i}^{j} & \\
\psi_{i}^{j}, \xi_{i} \geq 0 . &
\end{array}\right.
$$

The cost function in (4) contains a novel contextual term (made up of $N \cdot M$ elements) whose aim is to regularize the learning process with respect to the behavior of the context patterns in the neighborhood of the training pattern under consideration. The rationale of this term is to balance the contribution of possibly mislabeled training samples according to the semilabeled pixels of the neighborhood. The context slack variables $\psi_{i}^{j}=\psi_{i}^{j}\left(\tilde{\mathbf{x}}_{i}^{j}, \tilde{y}_{i}^{j}, \mathbf{w}, b\right)$ depend on $\tilde{\mathbf{x}}_{i}^{j} \in \Delta_{M}\left(\mathbf{x}_{i}\right)$ and, accordingly, permit one to directly take into account the contextual information in the learning phase. They are defined as

$$
\begin{aligned}
\psi_{i}^{j}=\max \left\{0,1-\tilde{y}_{i}^{j} \cdot\left[\mathbf{w} \cdot \Phi\left(\tilde{\mathbf{x}}_{i}^{j}\right)+b\right]\right\} \\
\forall i=1, \ldots, N, \quad \forall j=1, \ldots, M .
\end{aligned}
$$

The parameters $\kappa_{i}^{j} \in \mathbb{R}_{0}^{+}$weight the context patterns $\tilde{\mathbf{x}}_{i}^{j}$ depending on the agreement of their semilabels $\tilde{y}_{i}^{j}$ with that of the related training sample $y_{i}$. The hypothesis at the basis of the weighting system of the context patterns is that the pixels in the same neighborhood system have high probability to be associated to the same information class (i.e., the labels of the pixels are characterized by high spatial correlation). In particular, $\kappa_{i}^{j}$,s are defined as follows:

$$
\kappa_{i}^{j}=\left\{\begin{array}{lll}
\kappa_{1} & \text { if } & y_{i}=\tilde{y}_{i}^{j} \\
\kappa_{2} & \text { if } & y_{i} \neq \tilde{y}_{i}^{j}
\end{array}\right.
$$


where $\kappa_{1}$ and $\kappa_{2}$ are chosen from the user. The role of $\kappa_{1}$ and $\kappa_{2}$ is to define the importance of the context patterns. In particular, it is very important to define the ratios $C / \kappa_{i}, i=1,2$, which tune the weight of context patterns with respect to the patterns of the original training set. According to our hypothesis, in order to adequately penalize the mislabeled training patterns, it is suggested to fix $\kappa_{1} \geq \kappa_{2}$ as, in general, contextual patterns whose semilabels are in agreement with the label of the related training pattern should be considered more reliable than those whose semilabels are different. The selection of $\kappa_{1}$ and $\kappa_{2}$ can be simplified by fixing a priori the ratio $\kappa_{1} / \kappa_{2}=K$, thus focusing the attention only on $\kappa_{1}$ or on the ratio $C / \kappa_{1}$.

It is worth noting that the novel cost function defined in (4) maintains the important property of convexity of the cost function of the standard SVM. This allows us to solve the problem according to quadratic programming algorithms. By properly adjusting the Karush-Kuhn-Tucker conditions [i.e., the necessary and sufficient conditions for solving (5)], we derived the following dual bound maximization problem:

$$
\left\{\begin{array}{c}
\max _{\alpha, \beta}\left\{\sum_{i=1}^{N}\left(\alpha_{i}+\sum_{j=1}^{M} \beta_{i}^{j}\right)\right. \\
-\frac{1}{2} \sum_{i=1}^{N} \sum_{h=1}^{N}\left[\begin{array}{c}
y_{i} y_{h} \alpha_{i} \alpha_{h} k\left(\mathbf{x}_{i}, \mathbf{x}_{h}\right) \\
+2 y_{i} \alpha_{i} \sum_{j=1}^{M} \tilde{y}_{h}^{i} \beta_{h}^{i} k\left(\mathbf{x}_{i}, \tilde{\mathbf{x}}_{h}^{j}\right) \\
+\sum_{q=1}^{M} \sum_{j=1}^{M} \tilde{y}_{i}^{q} \tilde{y}_{h}^{j} \beta_{i}^{q} \beta_{h}^{j} k\left(\tilde{\mathbf{x}}_{i}^{q}, \tilde{\mathbf{x}}_{h}^{j}\right)
\end{array}\right] \\
\sum_{i=1}^{N}\left(\begin{array}{c}
\left.y_{i} \alpha_{i}+\sum_{j=1}^{M} \tilde{y}_{i}^{j} \beta_{i}^{j}\right)=0 \\
0 \leq \alpha_{i} \leq C \\
0 \leq \beta_{i}^{j} \leq \kappa_{i}^{j}
\end{array} \quad \forall j=1, \ldots, N\right.
\end{array}\right.
$$

where $\alpha_{i}$ and $r_{i}$ are the Lagrange multipliers associated with original training patterns, while $\beta_{i}^{j}$ and $s_{i}^{j}$ are the Lagrange multipliers associated with contextual patterns. The Lagrange multipliers $\alpha_{i}$ associated with the original labeled patterns are superiorly bounded by $C$ (they all have the same importance). The upper bound for the Lagrange multipliers $\beta_{i}^{j}$ associated with context patterns is $\kappa_{i}^{j}$, as it comes from (7). Once $\alpha_{i}$ and $\beta_{i}^{j}$ $(i=1, \ldots, N, j=1, \ldots, M)$ are determined, the generic pattern $\mathrm{x}$ belonging to the investigated image $\mathcal{I}$ can be classified according to the following decision function:

$$
\begin{array}{r}
\hat{y}=\operatorname{sgn}\left\{\sum_{i=1}^{N}\left[y_{i} \alpha_{i} k\left(\mathbf{x}_{i}, \mathbf{x}\right)+\sum_{j=1}^{M} \tilde{y}_{i}^{j} \beta_{i}^{j} k\left(\tilde{\mathbf{x}}_{i}^{j}, \mathbf{x}\right)\right]+b\right\} \\
\forall \mathbf{x}_{i} \in \mathcal{X}, \forall \tilde{\mathbf{x}}_{i}^{j} \in \tilde{\mathcal{X}}
\end{array}
$$

where, given $f(\mathbf{x})=\sum_{i=1}^{N}\left[y_{i} \alpha_{i} k\left(\mathbf{x}_{i}, \mathbf{x}\right)+\sum_{j=1}^{M} \tilde{y}_{i}^{j} \beta_{i}^{j} k\left(\tilde{\mathbf{x}}_{i}^{j}\right.\right.$, $\mathbf{x})]+b, b$ is chosen so that $y_{i} f\left(\mathbf{x}_{i}\right)=1$ for any $i$ with $0<\alpha_{i}<C$ and $\tilde{y}_{i}^{j} f\left(\tilde{\mathbf{x}}_{i}^{j}\right)=1$ for any $i$ and $j$ with $0<\beta_{i}^{j}<\kappa_{i}^{j}$.

It is worth noting that the proposed formulation could be empirically defined by considering different analytical forms for the kernels associated with the original training samples and the context patterns (composite kernel approach). From a general perspective, this would increase the flexibility of the method. However, as the training patterns and the context patterns are represented by the same feature vectors, the use of composite kernels (which would result in a further increase of the number of free parameters to set in the leaning of the classifier and, thus, in an increase of the computational cost required from the model-selection phase) does not seem useful.

\section{Multiclass Architecture}

Let us extend the binary $\mathrm{CS}^{4} \mathrm{VM}$ to the solution of multiclass problems. Let $\Omega=\left\{\omega_{1}, \ldots, \omega_{L}\right\}$ be the set of $L$ information classes that characterize the considered problem. As for the conventional SVM, the multiclass problem should be addressed with a structured architecture made up of binary classifiers. However, the properties of $\mathrm{CS}^{4} \mathrm{VM}$ lead to an important difference with respect to the standard supervised SVM. This difference is related to the step 2 of the learning of the $\mathrm{CS}^{4} \mathrm{VM}$. In this step, we assume to be able to give a reliable label to all patterns in the neighborhood system of each training pattern. In order to satisfy this constraint, we should define binary classification problems for each $\mathrm{CS}^{4} \mathrm{VM}$ included in the multiclass architecture characterized from an exhaustive representation of classes.

Let each $\mathrm{CS}^{4} \mathrm{VM}$ of the multiclass architecture solve a binary subproblem, where each pattern should belong to one of the two classes $\Omega_{A}$ or $\Omega_{B}$, defined as proper subsets of the original set of labels $\Omega$. The contextual semisupervised approach requires that, for each binary $\mathrm{CS}^{4} \mathrm{VM}$ of the multiclass architecture, there must be an exhaustive representation of all possible labels, i.e.,

$$
\Omega_{A} \cup \Omega_{B}=\Omega
$$

If (10) is not satisfied, some semilabels of context patterns $\tilde{\mathbf{x}}_{i}^{j}$ may not be represented in the binary subproblem and the context-sensitive semisupervised learning cannot be performed. According to this constraint, we propose to adopt a one-againstall (OAA) multiclass architecture, which is made up of $L$ parallel $\mathrm{CS}^{4} \mathrm{VM}$, as shown in Fig. 3 .

The $l$ th $\mathrm{CS}^{4} \mathrm{VM}$ solves a binary problem defined by the information class $\left\{\omega_{l}\right\} \in \Omega$ against all the others $\Omega-\left\{\omega_{l}\right\}$. In this manner, all the binary subproblems of multiclass architecture satisfy (10). The "winner-takes-all" rule is used for taking the final decision, i.e.,

$$
\hat{\omega}=\underset{i=1, \ldots, L}{\arg \max }\left\{f_{i}(\mathbf{x})\right\}
$$

where $f_{i}(\mathbf{x})$ represent the output of the $i$ th $\mathrm{CS}^{4} \mathrm{VM}$.

It is worth noting that other multiclass strategies that are commonly adopted with standard SVM (such as the oneagainst-one) [21] cannot be used with the proposed $\mathrm{CS}^{4} \mathrm{VM}$ as they do not satisfy (10). Nevertheless, other multiclass architectures could be specifically developed for the $\mathrm{CS}^{4} \mathrm{VM}$ approach, which should satisfy the constraint defined in (10). 


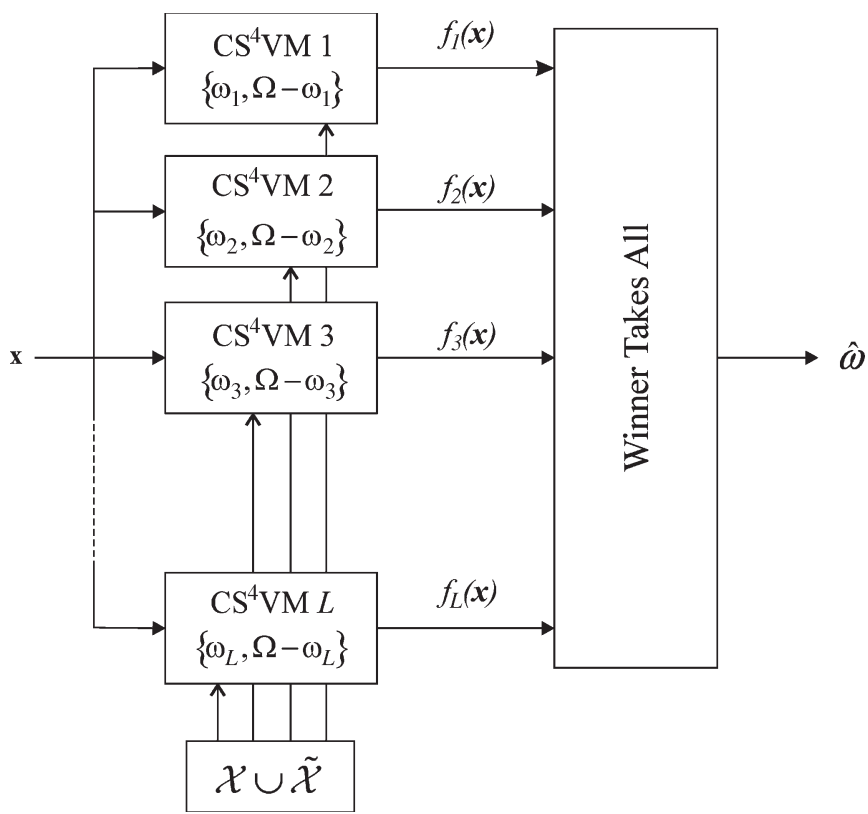

Fig. 3. OAA architecture for addressing the multiclass problem with the proposed $\mathrm{CS}^{4} \mathrm{VM}$.

\section{DESIGN OF EXPERIMENTS}

In this section, we describe the extensive experimental phase carried out to evaluate the robustness to the presence of noisy training samples of the proposed $\mathrm{CS}^{4} \mathrm{VM}$ and of other standard supervised and semisupervised classification algorithms. In particular, we compare the accuracy (in terms of kappa coefficient [22]) obtained by the proposed $\mathrm{CS}^{4} \mathrm{VM}$ with those yielded by other classification algorithms: the progressive semisupervised SVM $\left(\mathrm{PS}^{3} \mathrm{VM}\right)$ [17], the standard supervised SVM, the ML, and the $k$-NNs. We carried out different kinds of experiments by training the classifiers: 1) with the original training samples (with their correct labels) and 2) with different synthetic training sets, where mislabeled patterns (i.e., patterns with wrong labels) were added to the original training set in different percentages $(10 \%, 16 \%, 22 \%$, and $28 \%$ ) with respect to the total number of training samples. In the second kind of experiments, we manually introduced mislabeled training samples by considering the particular scene under investigation and simulating realistic mislabeling errors (e.g., caused by possible photointerpretation errors). The spatial location of wrong samples was distributed over the whole scene, by considering also clusters of pixels in the same neighborhood system. We analyzed the effects of noisy training sets on the classification accuracy in two different scenarios (which simulate different kinds of mislabeling errors): 1) Wrong samples are uniformly added to all the information classes (thus simulating the presence of mislabeling errors in the training points that does not depend on the land-cover type), and 2) wrong patterns are added to one specific class or to a subset of the considered classes (thus simulating a systematic error in the collection of ground truth samples for specific land-cover types).

In all the experiments, for the ML classifier, we adopted the Gaussian function as a model for the probability density functions of the classes. Concerning the $k$-NN classification algorithm, we carried out several trials, varying the value of $k$ from 1 to 40 in order to identify the value that maximizes the kappa accuracy on the test set.

For the SVM-based classifiers $\left(\mathrm{CS}^{4} \mathrm{VM}, \mathrm{PS}^{3} \mathrm{VM}\right.$, and standard SVM), we employed the sequential minimal optimization algorithm [23] (with proper modifications for the $\mathrm{CS}^{4} \mathrm{VM}$ ) and used Gaussian kernel functions (ruled by the free parameter $\sigma$ that expresses the width of the Gaussian function). All the data were normalized to a range $[0,1]$, and the model selection for deriving the learning parameters was carried out according to a grid-search strategy on the basis of the kappa coefficient of accuracy obtained on the test set.

For the standard SVM, the value of $2 \sigma^{2}$ was varied in the range $\left[10^{-2}, 10\right]$, while the values of $C$ were concentrated in the range [20, 200] after a first exploration in a wider range. For the model selection of both the $\mathrm{CS}^{4} \mathrm{VM}$ and the $\mathrm{PS}^{3} \mathrm{VM}$, we considered the same values for $C$ and $2 \sigma^{2}$ as for the SVM in order to have comparable results. Moreover, for the proposed $\mathrm{CS}^{4} \mathrm{VM}$, we fixed the value of $K=\kappa_{1} / \kappa_{2}=2$ and used the following values for $C / \kappa_{1}: 2,4,6,8,10,12$, and 14. For the definition of the context patterns, we considered a first-order neighborhood system. With regard to the $\mathrm{PS}^{3} \mathrm{VM}$, the value of $C^{*(0)}$ was varied in the range $[0.1,1]$, the one of $\gamma$ was varied in the range $[10,100]$, and $\rho$ was varied in the range $[10,100]$.

For simplicity, the model selection for all the SVM-based classifiers and the $k-\mathrm{NN}$ algorithm was carried out on the basis of the kappa coefficient of accuracy computed on the test set, which does not contain mislabeled samples. It is worth noting that this does not affect the relative results of the comparison, as the same approach was used for all the classifiers. It is important to observe that the proposed $\mathrm{CS}^{4} \mathrm{VM}$ method does not rely on the assumption of noise-free samples in the test set for parameter settings. The use of context patterns is effective in mitigating the bias effect introduced by noisy patterns even if the selected model is optimized on a noisy test set. In this condition, we may have an absolute decrease of classification accuracy, but the capability to mitigate the effects of wrong samples on the final classification result does not change.

In the experiments, we considered two data sets: The first one is made up of a very high geometrical resolution multispectral image acquired by the Ikonos satellite over the city of Ypenburg (The Netherlands); the second one is made up of a mediumresolution multispectral image acquired by the sensor TM of Landsat-5 in the surroundings of the city of Trento (Italy). The results obtained on the two data sets are presented in the following two sections.

\section{Experimental Results: IKonos Data SET}

The first considered data set is made up of the first three bands (corresponding to visible wavelengths) of an Ikonos subscene of size $387 \times 419$ pixels (see Fig. 4). The $4-m$ spatial resolution spectral bands have been reported to a $1-\mathrm{m}$ spatial resolution according to the Gram-Schmidt pansharpening procedure [24]. The available ground truth (which included 


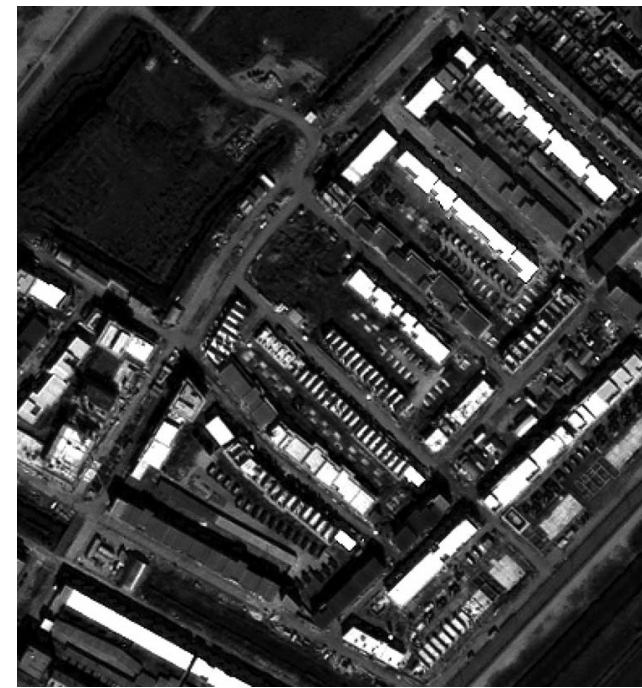

Fig. 4. Band 3 of the Ikonos image.

TABLE I

Number of PATTERNS IN THE TRAINING And Test Sets (IKONos Data SeT)

\begin{tabular}{|c|c|c|c|}
\hline \multirow{2}{*}{\multicolumn{2}{|c|}{ Class }} & \multicolumn{2}{|c|}{ Number of patterns } \\
\hline & & Training Set & Test Set \\
\hline & Grass & 63 & 537 \\
\hline & Road & 82 & 376 \\
\hline \multirow{4}{*}{ } & Small-aligned & 62 & 200 \\
\hline & White-roof & 87 & 410 \\
\hline & Gray-roof & 65 & 336 \\
\hline & Red-roof & 19 & 92 \\
\hline & Shadow & 30 & 231 \\
\hline
\end{tabular}

the information classes grass, road, shadow, small-aligned building, white-roof building, gray-roof building, and red-roof building) collected on two spatially disjoint areas was used to derive a training set and a test set for the considered image (see Table I). This setup allowed us to study the generalization capability of the systems by performing validation on areas spatially disjoint from those used in the learning of the classification algorithm. This is very important because of the nonstationary behavior of the spectral signatures of classes in the spatial domain. Starting from the original training set, several data sets were created by adding different percentages of mislabeled pixels in order to simulate noisy training sets as described in the previous section.

\section{A. Results With Mislabeled Training Patterns Uniformly Added to All Classes}

In the first set of experiments, different percentages (10\%, $16 \%, 22 \%$, and $28 \%$ ) of mislabeled patterns (with respect to the total number of samples) were uniformly added to all classes of the training set. The accuracy yielded on the test set by all the considered classifiers versus the percentage of mislabeled patterns are reported in Table II and shown in Fig. 5. As one can see, with the original training set, the proposed $\mathrm{CS}^{4} \mathrm{VM}$ exhibited a higher kappa coefficient of accuracy than the other classifiers. In greater detail, the kappa coefficient
TABLE II

Kappa Coefficient of Accuracy on the Test Set With Different PERCENTAGes of Mislabeled PatTERnS AdDED Uniformly to the Training Set (IKonos Data SeT)

\begin{tabular}{|c|c|c|c|c|c|}
\hline \multirow{2}{*}{$\begin{array}{c}\text { \% of mislabeled } \\
\text { patterns }\end{array}$} & \multicolumn{5}{|c|}{ Kappa Accuracy } \\
\cline { 2 - 6 } & $\mathbf{C S}^{\mathbf{4}} \mathbf{V M}$ & $\mathbf{P S}^{\mathbf{3}} \mathbf{V M}$ & $\mathbf{S V M}$ & $\boldsymbol{k}$-NN & ML \\
\hline 0 & 0.927 & 0.907 & 0.907 & 0.861 & 0.847 \\
\hline 10 & 0.919 & 0.910 & 0.907 & 0.803 & 0.688 \\
\hline 16 & 0.921 & 0.869 & 0.866 & 0.787 & 0.801 \\
\hline 22 & 0.893 & 0.862 & 0.861 & 0.781 & 0.727 \\
\hline 28 & 0.905 & 0.874 & 0.860 & 0.763 & 0.675 \\
\hline
\end{tabular}

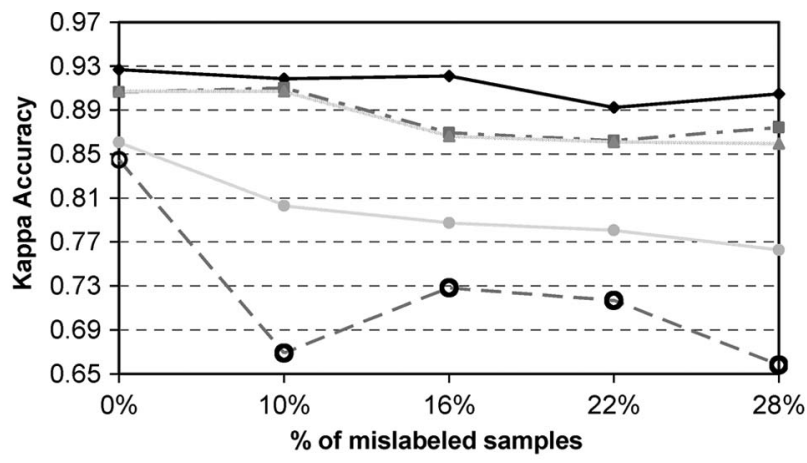

$\longrightarrow-\operatorname{CS} 4 \mathrm{VM}---\mathrm{PS} 3 \mathrm{VM} \backsim \mathrm{SVM} \longrightarrow \mathrm{k}-\mathrm{NN}-\boldsymbol{-} \longrightarrow \mathrm{ML}$

Fig. 5. Behavior of the kappa coefficient of accuracy on the test set versus the percentage of mislabeled training patterns uniformly distributed over all classes introduced in the training set (Ikonos data set).

obtained with the $\mathrm{CS}^{4} \mathrm{VM}$ is slightly higher than the ones obtained with the standard SVM and the $\mathrm{PS}^{3} \mathrm{VM}(+1.6 \%)$ and sharply higher than those yielded by the $k$-NN $(+6.6 \%)$ and the ML $(+8 \%)$. This confirms that the semisupervised exploitation of contextual information of training patterns allows us to increase the classification accuracy (also if their labels are correct). In this condition, the $\mathrm{PS}^{3} \mathrm{VM}$ classifier did not increase the classification accuracy of the standard SVM. When mislabeled samples were added to the original training set, the accuracies obtained with ML and $k$-NN classifiers sharply decreased, whereas SVM-based classifiers showed to be much more robust to "noise" (by increasing the number of mislabeled samples, the kappa accuracy decreased slowly). In greater detail, the kappa accuracy of the ML classifier decreased by $15.9 \%$ in the case of $10 \%$ of mislabeled samples with respect to the result obtained in the noise-free case, while the $k$-NN reduced its accuracy by $5.8 \%$ in the same condition. More generally, the $k$-NN classifier exhibited higher and more stable accuracies than the ML with all the considered amounts of noisy patterns. In all the considered trials, the proposed $\mathrm{CS}^{4} \mathrm{VM}$ exhibited higher accuracy than the other classifiers. In addition, with moderate and large numbers of mislabeled patterns $(16 \%, 22 \%$, and $28 \%)$, it was more stable than the SVM and the $\mathrm{PS}^{3} \mathrm{VM}$. In the trials with noisy training sets, the $\mathrm{PS}^{3} \mathrm{VM}$ classifier slightly increased the accuracy obtained by the standard SVM.

In order to better analyze the results of SVM and $\mathrm{CS}^{4} \mathrm{VM}$, we compared the average and the minimum kappa accuracies of the binary classifiers that made up the OAA multiclass architecture 


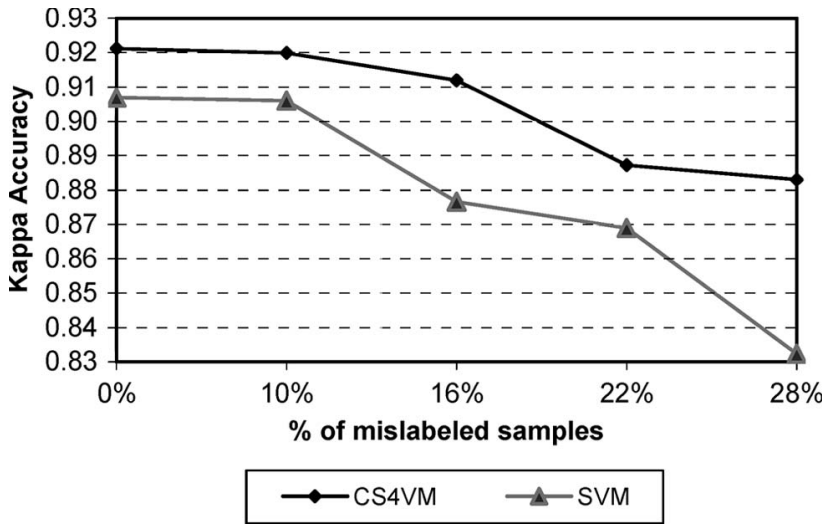

Fig. 6. Behavior of the average kappa coefficient of accuracy (computed on all the binary $\mathrm{CS}^{4} \mathrm{VMs}$ and SVMs included in the multiclass architecture) versus the percentage of mislabeled training patterns uniformly added to all classes (Ikonos data set).

TABLE III

KAPPA COEFFICIENT OF ACCURACY EXHIBITED FROM THE BINARY $\mathrm{CS}^{4}$ VM AND SVM THAT RESULTED IN THE LOWEST ACCURACY AMONG ALL BINARY ClasSIFIERS INCLUDED IN THE Multiclass ARChitecture Versus the Percentages OF Mislabeled TRAining PatTERnS UNiformly AdDed to All Classes (IKONOS Data SET)

\begin{tabular}{|c|c|c|c|}
\hline \% of mislabeled & \multicolumn{3}{|c|}{ Kappa Accuracy } \\
\cline { 2 - 4 } patterns & $\mathbf{C S}^{\mathbf{4}} \mathbf{V M}$ & $\mathbf{S V M}$ & $\boldsymbol{\Delta}(\mathbf{\%})$ \\
\hline 0 & 0.783 & 0.756 & 2.7 \\
\hline 10 & 0.784 & 0.767 & 1.8 \\
\hline 16 & 0.757 & 0.738 & 1.9 \\
\hline 22 & 0.751 & 0.691 & 6.0 \\
\hline 28 & 0.755 & 0.509 & 24.6 \\
\hline
\end{tabular}

(see Fig. 6 and Table III). It is possible to observe that the average kappa accuracy of the binary $\mathrm{CS}^{4} \mathrm{VMs}$ was higher than that of the binary SVMs and exhibited a more stable behavior when the amount of noise increased. Moreover, the accuracy of the class most affected by the inclusion of mislabeled patterns in the training set was very stable with the proposed classification algorithm, whereas it sharply decreased with the standard SVM when large percentages of mislabeled patterns were included in the training set. This confirms the effectiveness of the proposed $\mathrm{CS}^{4} \mathrm{VM}$, which exploits the contributions of the contextual term (and, thus, of contextual patterns) for mitigating the effects introduced by the noisy samples.

\section{B. Results With Mislabeled Training Patterns Concentrated on Specific Classes}

In the second set of experiments, several samples of the class "grass" were added to the original training set with the wrong label "road" in order to reach $10 \%$ and $16 \%$ of noisy patterns. In addition, "white-roof building" patterns were included with label "gray-roof building" to reach $22 \%$ and $28 \%$ of noisy samples. The resulting classification problem proved quite critical, as confirmed by the significant decrease in the kappa accuracies yielded by the considered classification algorithms (see Fig. 7 and Table IV). Nevertheless, also in this case, the context-based training of the $\mathrm{CS}^{4} \mathrm{VM}$ resulted in a significant

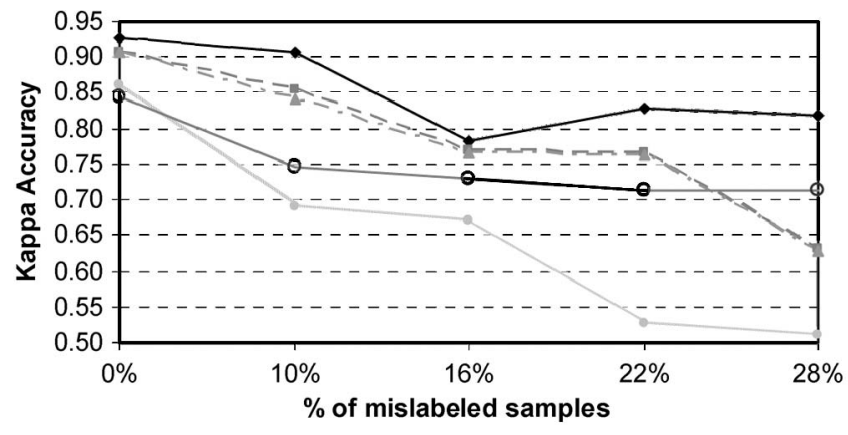

$\longrightarrow$ CS4VM $\longrightarrow--$ PS3VM $-\bullet--S V M \longrightarrow$ ML

Fig. 7. Behavior of the kappa coefficient of accuracy on test set versus the percentage of mislabeled training patterns concentrated on specific classes of the training set (Ikonos data set)

TABLE IV

Kappa Coefficient of ACCURACY ON The Test Set With Different Percentages of Mislabeled Patterns Added to Specific Classes of the Training Set (IKonos Data Set)

\begin{tabular}{|c|c|c|c|c|c|}
\hline \multirow{2}{*}{$\begin{array}{c}\text { \% of mislabeled } \\
\text { patterns }\end{array}$} & \multicolumn{5}{|c|}{ Kappa Accuracy } \\
\cline { 2 - 6 } & $\mathbf{C S}^{\mathbf{4}} \mathbf{V M}$ & $\mathbf{P S}^{\mathbf{3}} \mathbf{V M}$ & SVM & $\boldsymbol{k}$-NN & ML \\
\hline \hline 0 & 0.927 & 0.907 & 0.907 & 0.861 & 0.847 \\
\hline 10 & 0.906 & 0.855 & 0.841 & 0.690 & 0.746 \\
\hline 16 & 0.781 & 0.769 & 0.765 & 0.672 & 0.734 \\
\hline 22 & 0.828 & 0.767 & 0.762 & 0.525 & 0.722 \\
\hline 28 & 0.820 & 0.632 & 0.629 & 0.510 & 0.721 \\
\hline
\end{tabular}

increase of accuracy with respect to other classifiers. The kappa accuracy of the $k$-NN classifier dramatically decreased when the percentage of noisy patterns increased (in the specific case of $28 \%$ of mislabeled samples, the kappa accuracy decreased by $35.1 \%$ with respect to the original training set). The ML decreased its accuracy by $10.1 \%$, with $10 \%$ of noisy patterns, but exhibited a more stable behavior with respect to the $k$-NN when the amount of noisy patterns was further increased. The standard SVM algorithm obtained accuracies higher than those yielded by the $k$-NN and ML classifiers, while the $\mathrm{PS}^{3} \mathrm{VM}$ classifier, in general, slightly improved the accuracy of the standard SVM. However, with $28 \%$ of noisy patterns, the kappa accuracy sharply decreased to 0.629 (below the performance of ML). This behavior was strongly mitigated by the proposed $\mathrm{CS}^{4} \mathrm{VM}$ (which exhibited kappa accuracy of 0.820 in the same conditions).

Considering the behavior of the average kappa of the binary SVMs and $\mathrm{CS}^{4} \mathrm{VMs}$ that made up the OAA multiclass architecture (see Fig. 8), it is possible to note that the $\mathrm{CS}^{4} \mathrm{VM}$ always improved the accuracy of the standard SVM, and the gap between the two classifiers increased by increasing the amount of noisy samples. In the very critical case of $28 \%$ of mislabeled patterns, the context-based learning of $\mathrm{CS}^{4} \mathrm{VM}$ improved the average kappa accuracy of binary SVMs by $9.2 \%$. Moreover, the kappa coefficient of the class with the lowest accuracy with the proposed $\mathrm{CS}^{4} \mathrm{VM}$, even if small, was sharply higher than that of the standard SVM in all the considered trials (see Table V). This behavior shows that on this data set, the proposed method always improved the accuracy of the most critical binary classifier. 


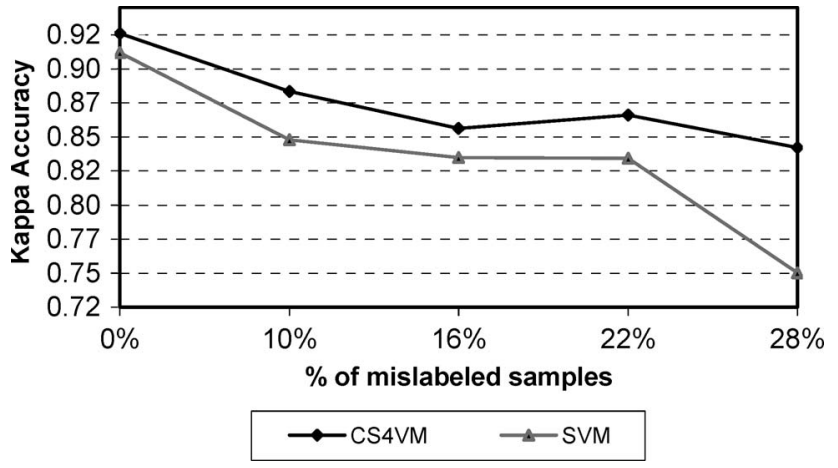

Fig. 8. Behavior of the average kappa coefficient of accuracy (computed on all the binary $\mathrm{CS}^{4} \mathrm{VMs}$ and SVMs included in the multiclass architecture) versus the percentage of mislabeled training patterns concentrated on specific classes (Ikonos data set).

TABLE V

KAPPA COEFFICIENT OF ACCURACY EXHIBITED FROM THE BINARY $\mathrm{CS}^{4}$ VM AND SVM THAT RESULTED IN THE LOWEST ACCURACY AMONG ALL BINARY ClASSIFIERS INCLUDED IN THE Multiclass Architecture Versus the Percentages of Mislabeled TRaining Patterns Concentrated on SPECIFIC Classes (IKonos DATA SET)

\begin{tabular}{|c|c|c|c|}
\hline \% of mislabeled & \multicolumn{3}{|c|}{ Kappa Accuracy } \\
\cline { 2 - 4 } patterns & $\mathbf{C S}^{\mathbf{4}} \mathbf{V M}$ & $\mathbf{S V M}$ & $\boldsymbol{\Delta}(\mathbf{\%})$ \\
\hline \hline 0 & 0.783 & 0.756 & 2.7 \\
\hline 10 & 0.620 & 0.422 & 19.8 \\
\hline 16 & 0.449 & 0.360 & 8.9 \\
\hline 22 & 0.538 & 0.360 & 17.8 \\
\hline 28 & 0.450 & 0.360 & 9.0 \\
\hline
\end{tabular}

Fig. 9 shows the classification maps obtained by training the considered classifiers with $28 \%$ of mislabeled patterns added on specific classes ("roads" and "gray-roof buildings") of the training set (the map obtained with the $\mathrm{PS}^{3} \mathrm{VM}$ is not reported because it is very similar to the one yielded with the SVM classifier). As one can see, in the classification maps obtained with the SVM, the $k$-NN, and the ML algorithms, many pixels of the class grass are confused with the class road, while white-roof buildings are confused with the gray-roof buildings. This effect is induced by the presence of noisy training samples affecting the aforementioned classes. In greater detail, the SVM classifier was unable to correctly recognize the red-roof buildings, while the $k$-NN technique often misrecognized the shadows present in the scene as red-roof buildings and white-roof buildings as gray-roof buildings. Moreover, the thematic map obtained with the $k$-NN is very noisy and fragmented (as confirmed by the low kappa coefficient of accuracy). The thematic map obtained with the proposed $\mathrm{CS}^{4} \mathrm{VM}$ clearly appears more accurate and less affected by the presence of mislabeled patterns.

\section{Experimental Results: Landsat Data SeT}

The second data set consists of an image acquired by the Landsat-5 TM sensor with a GIFOV of $30 \mathrm{~m}$. The considered image has size of $1110 \times 874$ pixels and was taken in the surrounding of the city of Trento (Italy) (see Fig. 10). A sixclass classification problem (with forest, water, urban, rock, fields, and grass classes) was defined according to the available ground truth collected on two spatially disjoint areas and used to derive the training and test sets (see Table VI). As for the Ikonos data set, this setup allowed us to study the generalization capability of the algorithms by classifying areas spatially disjoint from those used in the learning of the classifier. The important difference between this data set and the previous one consists in the geometric resolution, which in this case is significantly smaller than in the previous case ( $30 \mathrm{~m}$ versus $1 \mathrm{~m}$ ). Similar to the Ikonos data set, several noisy training sets were created by adding different amount of mislabeled pixels to the original data set: 1) with uniform distribution over the classes and 2) concentrated on a specific class.

\section{A. Results With Mislabeled Training Patterns Uniformly Added to All Classes}

Table VII shows the accuracies obtained in the first set of experiments, where mislabeled patterns were uniformly added to the information classes. Fig. 11 shows the behavior of the kappa accuracy versus the number of mislabeled patterns included in the training set for all the considered classifiers. It is possible to observe that with the noise-free training set, the proposed $\mathrm{CS}^{4} \mathrm{VM}$ led to the highest accuracy, slightly improving the kappa coefficient of standard SVM by $0.8 \%$. The ML classifier performed very well with the noise-free training set (the kappa accuracy was 0.923 ) but decreased its accuracy to 0.778 when only $10 \%$ of mislabeled patterns were introduced in the original training set, and its accuracy further decreased to 0.691 when the mislabeled samples reached $16 \%$. The $k$-NN classifier led to a lower accuracy than the ML in the absence of noise but showed to be less sensitive to noisy patterns uniformly added to the training set, thus exhibiting a more stable behavior. On the contrary, the SVM-based classification algorithms proved to be robust to the presence of mislabeled training samples. Indeed, the excellent generalization capability of the SVM led to even slightly increase the classification accuracy when a small amount of mislabeled patterns was added to the training set. The $\mathrm{PS}^{3} \mathrm{VM}$ algorithm resulted in a small improvement with respect to the SVM classifier in the trials where mislabeled samples were added to the training set. The kappa accuracy of the SVM classifier slightly decreased when the mislabeled samples exceeded $16 \%$, reducing its accuracy by $3 \%$ with respect to the noise-free case. In these cases, the proposed $\mathrm{CS}^{4} \mathrm{VM}$ further enhanced the robustness of SVM, leading to kappa accuracies that were always above 0.91 .

This behavior is confirmed by the analysis of the average and minimum kappa computed on the binary classifiers (see Fig. 12 and Table VIII), which highlights that the $\mathrm{CS}^{4} \mathrm{VM}$ significantly improved the accuracy with respect to the SVM. Such an improvement was more significant when increasing the amount of noise; thus, the $\mathrm{CS}^{4} \mathrm{VM}$ resulted in a more stable value of the kappa coefficient with respect to the percentage of mislabeled patterns present in the training set. It is worth noting that on this data set, the proposed $\mathrm{CS}^{4} \mathrm{VM}$ always improved the average kappa accuracy of the binary classifiers, even in cases where the global multiclass kappa coefficient of the $\mathrm{CS}^{4} \mathrm{VM}$ was slightly smaller than the one obtained with the standard SVM. This can be explained by observing that the decision strategy associated with the OAA multiclass architecture in some cases 


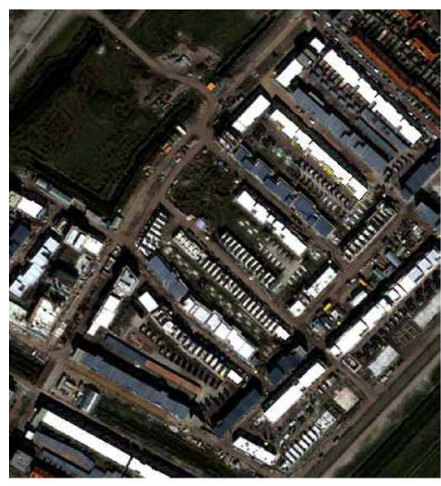

(a)

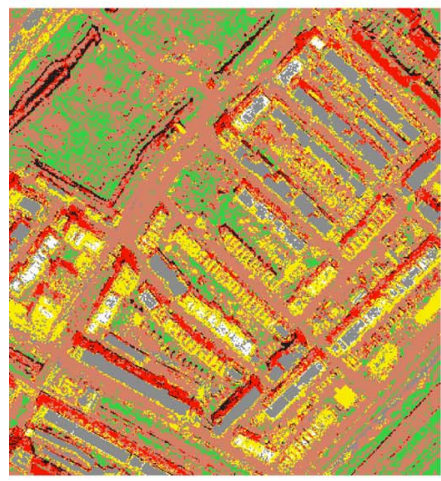

(d)

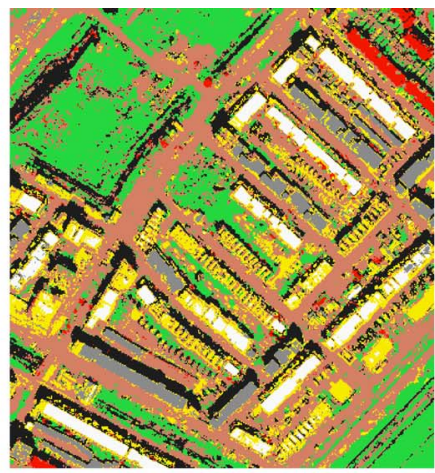

(b)

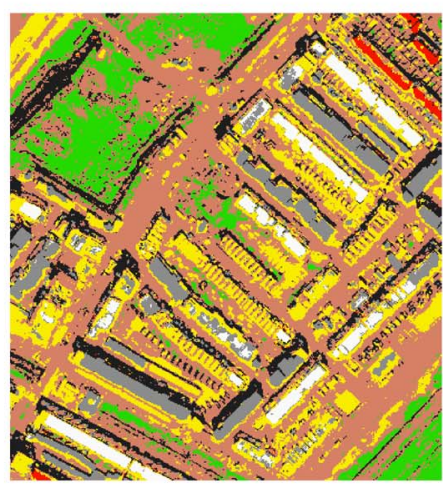

(e)

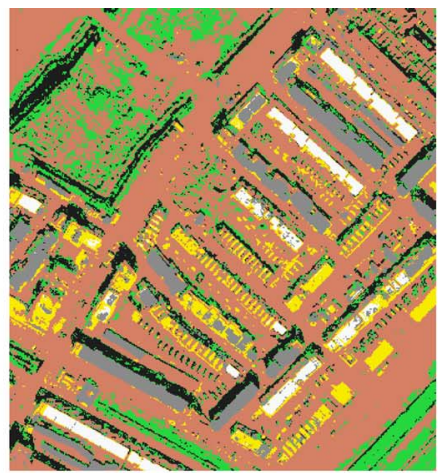

(c)

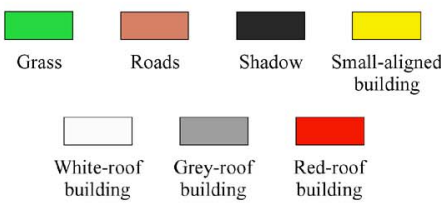

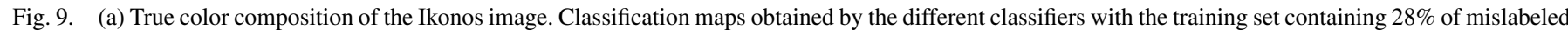
patterns added on specific classes. (b) $\mathrm{CS}^{4}$ VM. (c) SVM. (d) $k$-NN. (e) ML

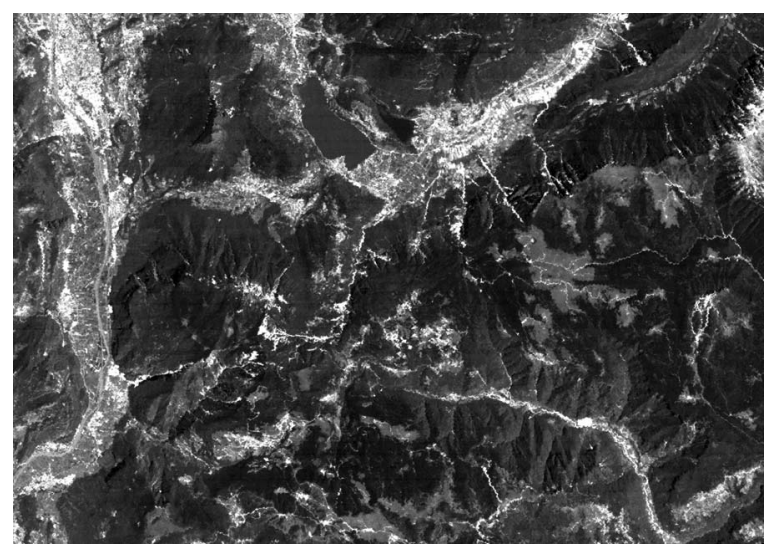

Fig. 10. Band 2 of the Landsat TM multispectral image.

TABLE VI

NUMBER OF PATTERNS IN THE TRAINING AND Test Set (Landsat Data SeT)

\begin{tabular}{|c|c|c|}
\hline \multirow{2}{*}{ Class } & \multicolumn{2}{|c|}{ Number of patterns } \\
\cline { 2 - 3 } & Training Set & Test Set \\
\hline \hline Forest & 128 & 538 \\
\hline Water & 118 & 177 \\
\hline Urban & 137 & 289 \\
\hline Rocks & 45 & 51 \\
\hline Fields & 93 & 140 \\
\hline Grass & 99 & 227 \\
\hline
\end{tabular}

TABLE VII

KAPPA COEFFICIENT OF ACCURACY ON TEST SET USING DifFERENT Percentages of Mislabeled Patterns AdDEd Uniformly to the Training Set (LANDSat Data Set)

\begin{tabular}{|c|c|c|c|c|c|}
\hline \multirow{2}{*}{$\begin{array}{c}\text { \% of mislabeled } \\
\text { patterns }\end{array}$} & \multicolumn{5}{|c|}{ Kappa Accuracy } \\
\cline { 2 - 6 } & $\mathbf{C S}^{\mathbf{4}} \mathbf{V M}$ & PS $^{\mathbf{3}} \mathbf{V M}$ & SVM & $\boldsymbol{k}$-NN & ML \\
\hline \hline 0 & 0.927 & 0.915 & 0.919 & 0.912 & 0.923 \\
\hline 10 & 0.930 & 0.935 & 0.931 & 0.905 & 0.778 \\
\hline 16 & 0.935 & 0.932 & 0.930 & 0.893 & 0.691 \\
\hline 22 & 0.921 & 0.891 & 0.886 & 0.868 & 0.686 \\
\hline 28 & 0.916 & 0.886 & 0.886 & 0.840 & 0.681 \\
\hline
\end{tabular}

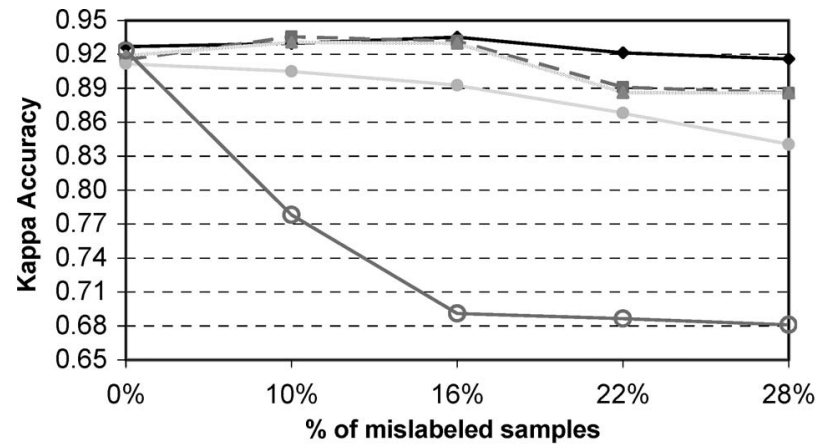

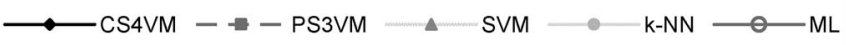

Fig. 11. Behavior of the kappa coefficient of accuracy on test set versus the percentage of mislabeled training patterns uniformly added to all classes (Landsat data set). 


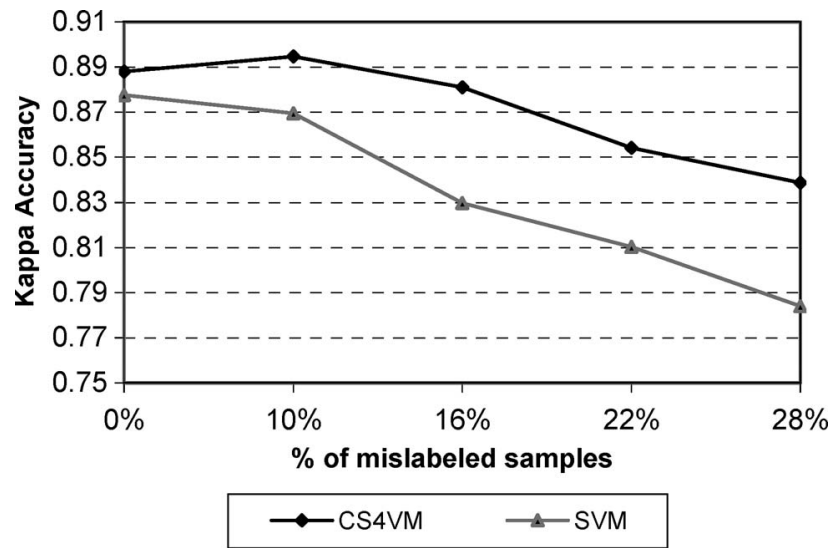

Fig. 12. Behavior of the average kappa coefficient of accuracy (computed on all the binary $\mathrm{CS}^{4} \mathrm{VMs}$ and SVMs included in the multiclass architecture) versus the percentage of mislabeled training patterns uniformly added to all classes (Landsat data set)

TABLE VIII

KAPPA COEFFICIENT OF ACCURACY EXHIBITED FROM THE CS ${ }^{4} \mathrm{VM}$ AND SVM THAT RESUlted IN THE LOWEST ACCURACY AMONG ALL BinARY Classifiers INCLUDED IN THE MULTICLASS ARCHITECTURE Versus the Percentages of Mislabeled Training Patterns Uniformly AdDED to All Classes (LANDSAT Data Set)

\begin{tabular}{|c|c|c|c|}
\hline \multirow{2}{*}{$\begin{array}{c}\text { \% of mislabeled } \\
\text { patterns }\end{array}$} & \multicolumn{3}{|c|}{ Kappa Accuracy } \\
\cline { 2 - 4 } & $\mathbf{C S}^{\mathbf{4}} \mathbf{V M}$ & $\mathbf{S V M}$ & $\boldsymbol{\Delta} \mathbf{( \% )}$ \\
\hline 0 & 0.701 & 0.701 & 0.0 \\
\hline 10 & 0.701 & 0.701 & 0.0 \\
\hline 16 & 0.650 & 0.627 & 2.3 \\
\hline 22 & 0.650 & 0.579 & 7.1 \\
\hline 28 & 0.641 & 0.498 & 14.3 \\
\hline
\end{tabular}

could "recover" the errors of binary classifiers by assigning the correct label to a pattern when comparing the output of binary classifiers. Nevertheless, the increased average accuracy of the binary $\mathrm{CS}^{4} \mathrm{VMs}$ is an important property because it involves more stable and reliable classification results.

Fig. 13 shows the classification maps obtained by training the classifiers with $28 \%$ of mislabeled patterns uniformly added to all the classes. It is possible to observe that the map generated by the proposed $\mathrm{CS}^{4} \mathrm{VM}$ is the most accurate. In the maps yielded by the SVM, the $k$-NN, and the ML algorithms, several pixels are misclassified as water (the map obtained with the $\mathrm{PS}^{3} \mathrm{VM}$ is not reported as very similar to the SVM map). In greater detail, the map obtained with the $k$-NN presents confusion between the classes water and urban, and the classes forest and water. In the map obtained by the ML, grass areas are often confused with forest.

\section{B. Results With Mislabeled Training Patterns Concentrated on a Specific Class}

In the second set of experiments, several samples of the class "forest" were added to the class "fields" to reach 10\%, 16\%, $22 \%$, and $28 \%$ of mislabeled patterns with respect to the total number of training samples. Moreover, in this case, the presence of errors that systematically affected one class severely impacted the performance of the supervised classification algorithms. When a low percentage $(10 \%)$ of noisy patterns was added to the original training set, all the considered classifiers decreased their kappa coefficient of accuracy by more than $12 \%$ (see Table IX and Fig. 14). In contrast to the first set of experiments, also the SVM algorithm suffered the presence of this type of noisy training set, reducing its accuracy by $18.4 \%$ (while the $k$-NN decreased its accuracy by $20.2 \%$ and the ML by $22.5 \%$ ). The semisupervised approach based on the $\mathrm{PS}^{3} \mathrm{VM}$ was not able to improve the accuracies of the standard SVM. The $\mathrm{CS}^{4} \mathrm{VM}$ could partially recover the accuracy of standard SVM by increasing the kappa accuracy by $7.4 \%$, thus limiting the effect of mislabeled patterns. When the amount of noisy patterns further increased, $\mathrm{PS}^{3} \mathrm{VM}, \mathrm{SVM}, \mathrm{ML}$, and $k$-NN classifiers did not further decrease significantly their kappa accuracies.

This behavior is confirmed from the average kappa coefficient of accuracy of the binary classifiers versus the percentage of mislabeled training patterns (see Fig. 15). In this case, we do not report the results of the binary classifiers exhibiting the lowest accuracy, because the complexity of the problem resulted in unreliable kappa values on this class (even if, also in this case, the $\mathrm{CS}^{4} \mathrm{VM}$ outperformed the SVM).

\section{DISCUSSION AND CONCLUSION}

In this paper, we have proposed a novel classification technique based on SVM that exploits the contextual information in order to render the learning of the classifier more robust to possible mislabeled patterns present in the training set. Moreover, we have analyzed the effects of mislabeled training samples on the classification accuracy of supervised algorithms, comparing the results obtained by the proposed $\mathrm{CS}^{4} \mathrm{VM}$ with those yielded by a $\mathrm{PS}^{3} \mathrm{VM}$, a standard supervised SVM, a Gaussian ML, and a $k$-NN. This analysis was carried out, varying both the percentage of mislabeled patterns and their distribution on the information classes. The experimental results obtained on two different data sets (a VHR image acquired by the Ikonos satellite and a medium-resolution image acquired by the Landsat-5 satellite) confirm that the proposed $\mathrm{CS}^{4} \mathrm{VM}$ approach exhibits augmented robustness to noisy training sets with respect to all the other classifiers. In greater detail, the proposed $\mathrm{CS}^{4} \mathrm{VM}$ method always increased the average kappa coefficient of accuracy of the binary classifiers included in the OAA multiclass architecture with respect to the standard SVM classifier. Moreover, in many cases, the $\mathrm{CS}^{4} \mathrm{VM}$ sharply increased the accuracy on the information class that was most affected by the mislabeled patterns introduced in the training set.

By analyzing the effects of the distribution of mislabeled patterns on the classes, it is possible to conclude that errors concentrated on a class (or on a subset of classes) are much more critical than errors uniformly distributed on all classes. In greater detail, when noisy patterns were added uniformly to all classes, we observed that the proposed $\mathrm{CS}^{4} \mathrm{VM}$ resulted in higher and more stable accuracies than all the other classifiers. The supervised SVM and the $\mathrm{PS}^{3} \mathrm{VM}$ exhibited relatively high accuracies when a moderate amount of noisy patterns was included in the training set, but they slowly decreased their accuracy when the percentage of mislabeled samples increased. On the contrary, both the ML and the $k$-NN classifiers are very sensitive even to the presence of a small amount of noisy 


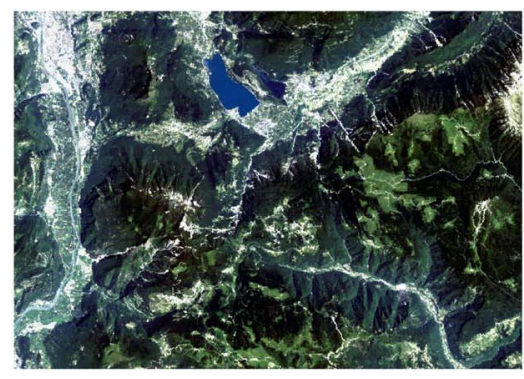

(a)

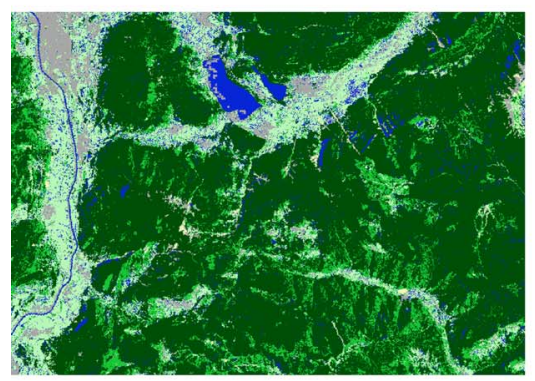

(d)

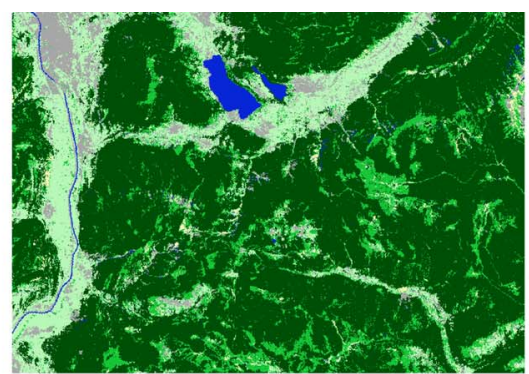

(b)

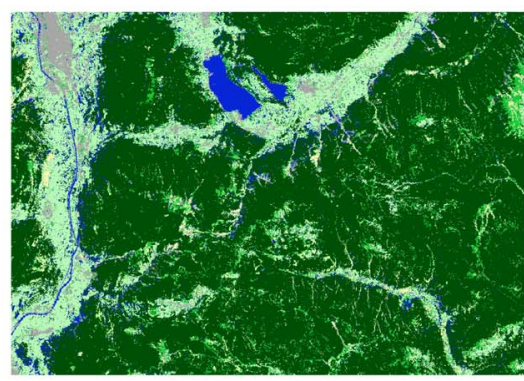

(e)

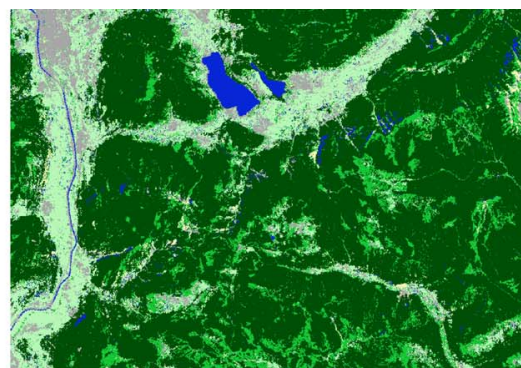

(c)

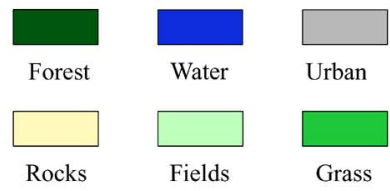

Fig. 13. (a) True color composition of Landsat image. Classification maps obtained by the different classifiers with the training set containing $28 \%$ of noisy patterns uniformly added to all classes. (b) $\mathrm{CS}^{4}$ VM. (c) SVM. (d) $k$-NN. (e) ML.

TABLE IX

Kappa Coefficient of Accuracy on the Test Set Using Training Sets With Different Percentages of Mislabeled Patterns AdDed to a SPECific Class (LANDSAT Data SET)

\begin{tabular}{|c|c|c|c|c|c|}
\hline \multirow{2}{*}{$\begin{array}{c}\text { \% of mislabeled } \\
\text { patterns }\end{array}$} & \multicolumn{5}{|c|}{ Kappa Accuracy } \\
\cline { 2 - 6 } & $\mathbf{C S}^{\mathbf{4}} \mathbf{V M}$ & $\mathbf{P S}^{\mathbf{3}} \mathbf{V M}$ & $\mathbf{S V M}$ & $\boldsymbol{k}$-NN & ML \\
\hline 0 & 0.927 & 0.915 & 0.919 & 0.882 & 0.923 \\
\hline 10 & 0.809 & 0.738 & 0.735 & 0.680 & 0.699 \\
\hline 16 & 0.712 & 0.706 & 0.695 & 0.652 & 0.678 \\
\hline 22 & 0.691 & 0.664 & 0.661 & 0.632 & 0.671 \\
\hline 28 & 0.658 & 0.651 & 0.648 & 0.632 & 0.666 \\
\hline
\end{tabular}

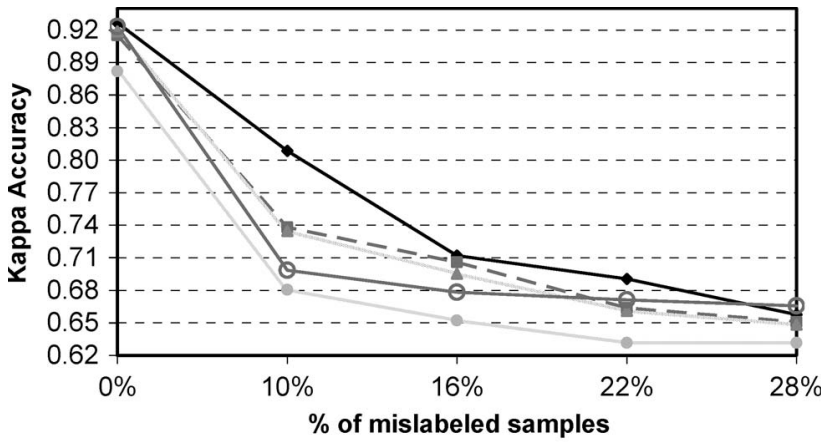

$\longrightarrow$ CS4VM - -

Fig. 14. Behavior of the kappa coefficient of accuracy on test set versus the percentage of mislabeled training patterns concentrated on a specific class (Landsat data set).

patterns and sharply decreased their accuracies by increasing the number of mislabeled samples. Nevertheless, the $k$-NN classifier resulted significantly more accurate than the ML classifier when mislabeled patterns equally affected the considered information classes. When noisy patterns were concentrated on a specific class of the training set, the accuracies of all the considered classifiers sharply decreased by increasing the

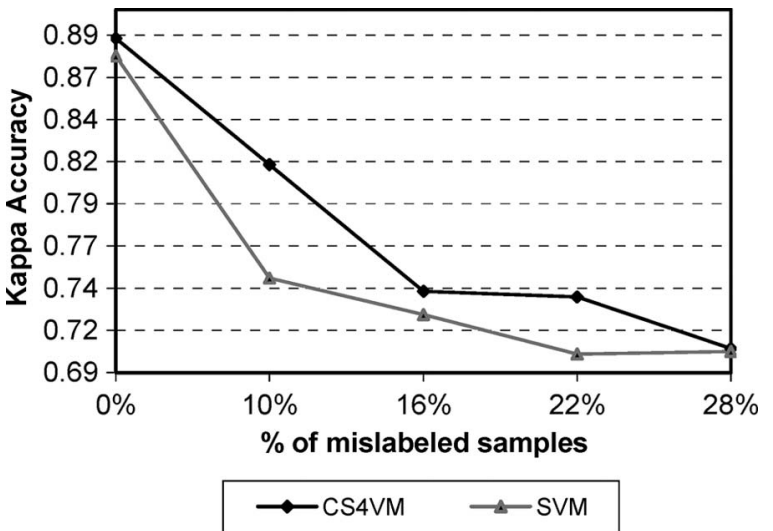

Fig. 15. Behavior of the average kappa coefficient of accuracy (computed on all the binary $\mathrm{CS}^{4} \mathrm{VMs}$ and SVMs included in the multiclass architecture) versus the percentage of mislabeled training patterns concentrated on a specific class (Landsat data set).

amount of mislabeled training samples. Moreover, in this case, the proposed $\mathrm{CS}^{4} \mathrm{VM}$ exhibited, in general, the highest and more stable accuracies. Nonetheless, when the number of mislabeled patterns increased over a given threshold, the classification problem became very critical and also the proposed technique significantly reduced its effectiveness. The standard SVM classifier still maintained higher accuracies than the ML and the $k$-NN techniques. The $\mathrm{PS}^{3} \mathrm{VM}$ slightly increased the accuracies of the standard SVM. Unlike the previous case, the $k$-NN algorithm resulted in lower accuracies than the ML method. This is mainly due to the fact that mislabeled patterns concentrated on a single class (or on few classes) alter the prior probabilities, thus affecting more the $k$-NN classifier (which implicitly considers the prior probabilities in the decision rule) than the ML technique (which does not consider the prior probabilities of classes). 
The proposed $\mathrm{CS}^{4} \mathrm{VM}$ introduces some additional free parameters with respect to the standard supervised SVM, which should be tuned in the model-selection phase. The analysis on the effects of the values of these parameters on the classification results (carried out in the different simulations described in this paper) pointed out that the empirical selection of $K=\kappa_{1} / \kappa_{2}=2$ (which is reasonable, considering the physical meaning of this ratio) resulted in good accuracies on both data sets. This choice allows one to reduce the model-selection phase to tune the value of the ratio $C / \kappa_{1}$ in addition to the standard SVM parameters. Nonetheless, when possible, the inclusion of the choice of the $\kappa_{1} / \kappa_{2}$ value in the model selection would optimize the results achievable with the proposed approach. The optimal value for the ratio $C / \kappa_{1}$ depends on the considered data set and the type of mislabeling errors, but in general, we observed that higher weights for the context patterns (lower values for the ratio $C / \kappa_{1}$ ) can result in better classification accuracies when the percentage of mislabeled training patterns increases. This confirms the importance of the context term to increase the classification accuracy in the presence of noisy training sets.

It is worth noting that the considered $\mathrm{PS}^{3} \mathrm{VM}$ classifier slightly improved the accuracy with respect to the standard SVM by exploiting the information of unlabeled samples, but it could not gain in accuracy when the amount of mislabeled patterns increased. Indeed, the $\mathrm{PS}^{3} \mathrm{VM}$ is not developed to take into account the possible presence of mislabeled training patterns, which affect the first iteration of the learning phase propagating the errors to the semilabeled samples in the next iterations of the algorithm. On the contrary, the proposed $\mathrm{CS}^{4} \mathrm{VM}$ is particularly developed to cope with "non fully reliable" training sets by exploiting the information of pixels in the neighborhood of the training points according to a specific weighting mechanism that penalizes less reliable training patterns. In addition, the proposed $\mathrm{CS}^{4} \mathrm{VM}$ approach is computationally less demanding than the $\mathrm{PS}^{3} \mathrm{VM}$ as it requires only two steps (this choice is done for limiting the computational complexity and is supported from empirical experiments that confirmed that increasing the number of iterations does not significantly change the classification results). On the contrary, the $\mathrm{PS}^{3} \mathrm{VM}$ may require a large number of iterations before convergence.

The computational cost of the learning phase of the proposed $\mathrm{CS}^{4} \mathrm{VM}$ method is slightly higher than that required from the standard supervised SVM. This depends on both the second step of the learning algorithm (which involves an increased number of samples, as semilabeled context patterns are considered in the process) and the setting of the additional parameters in the model-selection phase. In our experiments on the Ikonos data set, carried out on a PC mounting an Intel Pentium D processor at $3.4 \mathrm{GHz}$ and a 2-Gb DDR2 RAM, the training phase of a supervised SVM took in average about $20 \mathrm{~s}$, while the one of the proposed $\mathrm{CS}^{4} \mathrm{VM}$ required about $3 \mathrm{~min}$. It is important to point out that the additional cost of the proposed method concerns only the learning phase, whereas the computational time in the classification phase remains unchanged.

As a final remark, it is worth stressing that the proposed analysis points out the dramatic effects involved on the classi- fication accuracy from a relatively small percentages of mislabeled training samples concentrated on a class (or on a subset of classes). This should be understood in order to define adequate strategies in the design of training data for avoiding this kind of errors.

\section{REFERENCES}

[1] R. O. Duda, P. E. Hart, and D. G. Stork, Pattern Classification, 2nd ed. New York: Wiley, 2001.

[2] J. A. Richards and X. Jia, Remote Sensing Digital Image Analysis, 4th ed. Berlin, Germany: Springer-Verlag, 2006.

[3] M. Chi and L. Bruzzone, "An ensemble-driven k-NN approach to ill posed classification problems," Pattern Recognit. Lett., vol. 27, no. 4, pp. 301307, Mar. 2006.

[4] V. N. Vapnik, The Nature of Statistical Learning Theory, 2nd ed. New York: Springer-Verlag, 2001.

[5] C. Burges, "A tutorial on support vector machines for pattern recognition," Data Mining Knowl. Discovery, vol. 2, no. 2, pp. 121-167, Jun. 1998.

[6] N. Cristianini and J. Shawe-Taylor, An Introduction to Support Vector Machines and Other Kernel-Based Learning Methods. Cambridge, U.K.: Cambridge Univ. Press, 1995.

[7] B. Schölkopf and A. Smola, Learning With Kernels. Cambridge, MA: MIT Press, 2002. [Online]. Available: http://www.learning-withkernels.org/

[8] F. Melgani and L. Bruzzone, "Classification of hyperspectral remote sensing images with support vector machines," IEEE Trans. Geosci. Remote Sens., vol. 42, no. 8, pp. 1778-1790, Aug. 2004.

[9] G. F. Hughes, "On the mean accuracy of statistical pattern recognition," IEEE Trans. Inf. Theory, vol. IT-14, no. 1, pp. 55-63, Jan. 1968.

[10] F. Bovolo and L. Bruzzone, "A context-sensitive technique based on support vector machines for image classification," in Proc. IEEE PReMI, Kolkata, India, Dec. 2005, vol. 3776, pp. 260-265.

[11] A. A. Farag, R. M. Mohamed, and A. El-Baz, "A unified framework for MAP estimation in remote sensing image segmentation," IEEE Trans. Geosci. Remote Sens., vol. 43, no. 7, pp. 1617-1634, Jul. 2005.

[12] F. Melgani and S. Serpico, "A Markov random field approach to spatiotemporal contextual image classification," IEEE Trans. Geosci. Remote Sens., vol. 41, no. 11, pp. 2478-2487, Nov. 2003.

[13] G. Moser, S. Serpico, and F. Causa, "MRF model parameter estimation for contextual supervised classification of remote-sensing images," in Proc. IEEE IGARSS, Jul. 2005, pp. 308-311.

[14] P. Gamba, F. Dell'Acqua, G. Lisini, and G. Trianni, "Improved VHR urban area mapping exploiting object boundaries," IEEE Trans. Geosci. Remote Sens., vol. 45, no. 8, pp. 2676-2682, Aug. 2007.

[15] M. Berthod, Z. Kato, S. Yu, and J. Zerubia, "Bayesian image classification using Markov random fields," Image Vis. Comput., vol. 14, no. 4, pp. 285 295, May 1996.

[16] R. Nishii, "A Markov random field-based approach to decision-level fusion for remote sensing image classification," IEEE Trans. Geosci. Remote Sens., vol. 41, no. 10, pp. 2316-2319, Oct. 2003.

[17] L. Bruzzone, M. Chi, and M. Marconcini, "Semisupervised support vector machines for classification of hyperspectral remote sensing images," in Hyperspectral Data Exploitation, C.-I. Chang, Ed. Hoboken, NJ: Wiley, 2007, ch. 11, pp. 275-311.

[18] L. Bruzzone, M. Chi, and M. Marconcini, "A novel transductive SVM for semisupervised classification of remote-sensing images," IEEE Trans. Geosci. Remote Sens., vol. 44, no. 11, pp. 3363-3373, Nov. 2006.

[19] M. M. Dundar and D. A. Landgrebe, "A cost-effective semisupervised classifier approach with kernels," IEEE Trans. Geosci. Remote Sens., vol. 42, no. 1, pp. 264-270, Jan. 2004.

[20] K. P. Bennett and A. Demiriz, "Semi-supervised support vector machines," in Advances in Neural Information Processing Systems, vol. 10. Cambridge, MA: MIT Press, 1998, pp. 368-374.

[21] C. Hsu and C. Lin, "A comparison of methods for multi-class support vector machines," IEEE Trans. Neural Netw., vol. 13, no. 2, pp. 415-425, Mar. 2002.

[22] R. G. Congalton and K. Green, Assessing the Accuracy of Remotely Sensed Data. Boca Raton, FL: Lewis Publishers, 1999.

[23] J. Platt, "Fast training of support vector machines using sequential minimal optimization," in Advances in Kernel Methods: Support Vector Learning, B. Schölkopf, C. Burges, and A. Smola, Eds. Cambridge, MA: MIT Press, 1998, ch. 12, pp. 185-208.

[24] B. Aiazzi, S. Baronti, M. Selva, and L. Alparone, "Enhanced GramSchmidt spectral sharpening based on multivariate regression of MS and pan data," in Proc. IEEE IGARSS, 2006, pp. 3806-3809. 


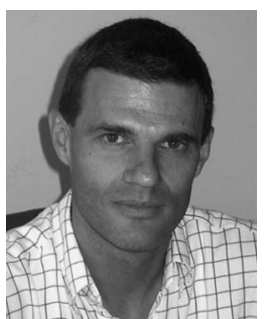

Lorenzo Bruzzone (S'95-M'98-SM'03) received the Laurea (M.S.) degree (summa cum laude) in electronic engineering and the Ph.D. degree in telecommunications from the University of Genoa, Genoa, Italy, in 1993 and 1998, respectively.

From 1998 to 2000, he was a Postdoctoral Researcher with the University of Genoa. Since 2000, he has been with the University of Trento, Trento, Italy, where he his currently a Full Professor of telecommunications. He teaches remote sensing, pattern recognition, and electrical communications. He is the Head of the Remote Sensing Laboratory in the Department of Information and Communication Technology, University of Trento. He conducts and supervises research on these topics within the frameworks of several national and international projects. He is an Evaluator of project proposals for many different governments (including European Commission) and scientific organizations. $\mathrm{He}$ is the author (or coauthor) of 60 scientific publications in referred international journals, more than 120 papers in conference proceedings, and seven book chapters. He is a Referee for many international journals and has served on the scientific committees of several international conferences. His current research interests are in the area of remote-sensing image processing and recognition (analysis of multitemporal data, feature extraction and selection, classification, regression and estimation, data fusion, and machine learning).

Dr. Bruzzone is a member of the Managing Committee of the Italian InterUniversity Consortium on Telecommunications and a member of the Scientific Committee of the India-Italy Center for Advanced Research. He is also a member of the International Association for Pattern Recognition and of the Italian Association for Remote Sensing. He was the General Chair and Cochair of the First and Second IEEE International Workshop on the Analysis of Multitemporal Remote-Sensing Images (MultiTemp) and is currently a member of the Permanent Steering Committee of this series of workshops. Since 2003, he has been the Chair of the SPIE Conference on Image and Signal Processing for Remote Sensing. From 2004 to 2006, he served as an Associated Editor of the IEEE Geoscience AND Remote Sensing LetTers, and currently, he is an Associate Editor of the IEEE TRANSACTIONS ON GEOSCIENCE AND REMOTE SEnsing. He ranked first place in the Student Prize Paper Competition of the 1998 IEEE International Geoscience and Remote Sensing Symposium (Seattle, July 1998). He was a recipient of the Recognition of IEEE TRANSACTIONS ON Geoscience ANd Remote SENSING Best Reviewers in 1999 and was a Guest Editor of a Special Issue of the IEEE TRANSACTIONS ON GEOSCIENCE AND REMOTE SENSING on the subject of the analysis of multitemporal remotesensing images (November 2003).

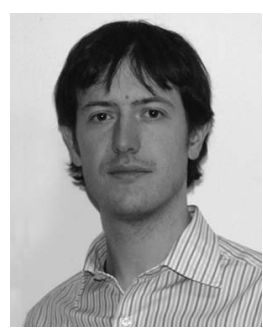

Claudio Persello (S'07) received the Laurea (B.S.) and Laurea Specialistica (M.S.) degrees in telecommunication engineering from the University of Trento, Trento, Italy, in 2003 and 2005, respectively, where he is currently working toward the $\mathrm{Ph} . \mathrm{D}$. degree in information and communication technologies.

$\mathrm{He}$ is working with the Remote Sensing Group at the Department of Information Engineering and Computer Science, University of Trento. His current research interests are in the area of remote sensing, image classification, pattern recognition, and machine learning.

Mr. Persello is a referee for the Canadian Journal of Remote Sensing and the iEEE Transactions on Geoscience and Remote Sensing. 